\title{
Remote sensing detection techniques for brownfield identification and monitoring by GIS tools
}

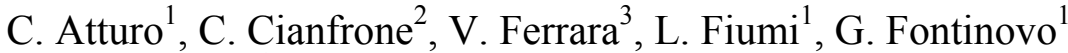 \\ \& C. M. Ottavi ${ }^{3}$ \\ ${ }^{1}$ LARA, IIA CNR, Area della Ricerca di Roma 2 Tor Vergata, Italy \\ ${ }^{2}$ Faculty of Engineering, University of Rome "La Sapienza", Italy \\ ${ }^{3}$ Department of Electronic Engineering, \\ University of Rome "La Sapienza", Italy
}

\begin{abstract}
This paper examines remote sensing detection techniques for data integration in GIS to be employed in brownfield monitoring. Brownfields are frequently associated with urban wastes, mostly from building demolition. The hazard increases when dangerous materials are used. In order to allow us to perform a detailed classification of the land and to identify a wide range of the brownfields, the Laboratory of CNR (LARA) detects environmental data by means of the airborne MIVIS (Multispectral Infrared and Visible Imaging Spectrometer) sensor. Moreover, GIS tools correlate the relevant data in order to evaluate the extension and derivative parameters of the same brownfields. These parameters are then used to elaborate indicators that can be used within the framework of a sustainable planning and development.

Keywords: monitoring of contaminated sites, multispectral sensors, MIVIS, GIS, multimedia modelling.
\end{abstract}

\section{Introduction to brownfield identification}

Once thriving areas of economic activity, brownfields are today generally abandoned/underused urban and sub-urban zones. Their expansion can be risky because it frequently involves some sort of environmental contamination, depending on the wear state of the building taken into consideration. They 
unavoidably create an empty architectural space with environmental and urban concerns. Brownfield sites, especially if urbanized (that is, with the utility networks still in place), and if well-linked, can be restored to productive use both from an environmental and economic viewpoint, thanks to the lower soil use .

The remote sensing classification techniques allow us to identify areas particularly at risk. The process of mapping of the roofing material and the detection of hazardous substances both in rural and in urban areas, represent the first and most important step to assess the risk level related to the study area under examination. As a matter of fact, these detected substances, should be at first considered as potentially hazardous only. The wear state, the maintenance and the repair of a building structure could reduce the related risk level. The interpretation of remotely sensed data must always be associated to in situ measurements because they are often decisive to distinguish among different kinds of situation and to provide a more accurate risk level assessment. These techniques, however, can considerably reduce the human resource employment and the related costs because they require a far less extensive ground campaign care must be taken however to validate the resulting risk level with reliable data.

When hazardous substances and materials are detected, a cross-verification technique should follow, i.e. a correlation among different territorial parameters or among indicators. It is evident that a substance becomes more hazardous when it is closer to other elements or buildings emphasizing their diffusive and explosive characteristics. Soluble substances detected close to springs, streams or simple canals, because of torrential phenomena, cannot be treated as those away from any element emphasizing their diffusive characteristics.

The main characterization require for brownfields often concerns the abandoned areas and their wear state. The presence of hazardous substances in the same area makes the situation even worse. A specific indicator should be provided in order to suggest, according to the risk level, the restoration monitoring as a priority. Within the framework of the urban areas, the Public Authorities entrusted of environmental monitoring can easily implement the recognition as well as the identification of wear state conditions. This thanks to the easy access to the area and to the protest of those who live close to brownfields. In these areas the automated or semi-automated techniques can, if this is the case, help identifying areas at risk. Whereas the same techniques can prove to be significantly more favourable for those less populated (or harder to access areas with recent wear state situation (often in rapid evolution).

The hyperspectral remote sensing, already intended for the territory monitoring and the relative qualification, provides essential data for further territorial classification. Basically all the pixels associated to the classified material are tagged with a brownfield label The pure recognition would make desirable a series of consecutive and derivative steps: extension evaluation, possible risk assessment according to the above-mentioned specifications, nearness evaluations and so on. All these steps have to be implemented by means of an appropriate GIS. The above automated or semi-automated techniques are implemented by the post-processing image coming from airborne remote sensing (in this case, based on hyperspectral data). These images are correlated, if 
needed, to others originating from a different source, geo-referred as the first ones and so overlaid over the same area even if having a different resolution. This could provide a more detailed recognition and a lower uncertainty of the geometric features.

\section{Brownfields: hyperspectral classification techniques}

To support the research activity, the Italian National Research Council has established the LARA Project (Airborne Laboratory for Environmental Research) with the aim to remotely collect data by means of an airborne platform system called MIVIS. It is a modular scanning system that operates with a high spectral and geometric resolution. It is composed of 4 spectrometers able to simultaneously collect samples in 102 spectral bands within $0.43 \mu \mathrm{m}$ and $12.7 \mu \mathrm{m}$. This allows the study of both the chemical and physical parameters of the Earth's surface. The MIDAS (Multispectral Interactive Data Processing and Analysis System) station converts data at ground and it is capable of performing the radiometric correction. The hyperspectral data processing and classification are aimed at characterizing several ground features of general interest such as building structures, road paving, crops, bare soils, vegetation, wastes and so on.

Table 1: $\quad$ MIVIS sensor characteristics.

\begin{tabular}{|c|l|c|c|c|}
\hline Spectrometers & Spectrum & Bands & $\begin{array}{c}\text { Spectral interval } \\
(\mu \mathrm{m})\end{array}$ & $\begin{array}{c}\text { Bandwidth } \\
(\mu \mathrm{m})\end{array}$ \\
\hline I & Visible & 20 & $0.43-0.83$ & 0.02 \\
\hline II & Near Infrared & 8 & $1.15-1.55$ & 0.06 \\
\hline III & Mean Infrared & 64 & $2.0-2.5$ & 0.009 \\
\hline IV & Thermal Infrared & 10 & $8.2-12.7$ & $0.34-0.54$ \\
\hline
\end{tabular}

The discrimination of waters and, as earlier mentioned, the identification of the characteristics related to abandoned or otherways investigated areas is based on the spectral signature concept: all the natural and artificial objects have different emission properties within the various optical bands used; they also exhibit and different values of backscattering from different radio waves. As a consequence, modern remote sensing systems are implemented taking into account the possibility of varying the observation parameters: the object is observed through different sensors varying the frequency, polarization and the incidence angle of the scenes. An appropriate integration of these hyperspectral data will follow, allowing us to extrapolate information otherwise not visible on data taken from single measures. Once acquired the raw data, a pre-processing phase is required. During this phase there is an attempt to reduce the following process of the scenes due to alignment problems of the different operating sensors; contrasts are altered or emphasized as needed, and the effects of high frequency attenuation are compensated. In 
addition, operations such as geometrical and co-ordinate transformations can be performed. Finally, the correction of atmospheric effects together with $I R C$ (Internal Radiometric Calibration) are implemented: the retro-diffused energy coming from the atmosphere towards the sensor can be considerably important. The radiometrically corrected is then processed using the supervised classification technique called Spectral Angle Mapper (SAM). This method enables us to quickly map the similarities existing between image spectra and reference spectra [2]. The reference spectra can be determined either in laboratory or in field; otherwise they can be extracted from images, (see Figure 1). The algorithm gives the spectral similarity between the two spectra in terms of the "angle" which they form, and, therefore, the spectra are considered as vectors in a space whose dimension is equal to the $n b$ (number of bands). The similarity between an unknown spectrum $t$ and a reference spectrum $r$ comes from the following equation:

$$
\alpha=\cos ^{-1}\left(\frac{\vec{t} \bullet \vec{r}}{\|\vec{t}\| \bullet\|\vec{r}\|}\right)
$$

that can also be expressed as follows:

$$
\alpha=\cos ^{-1}\left(\frac{\sum_{i=1}^{n b} t_{i} r_{i}}{\left(\sum_{i=1}^{n b} t_{i}^{2}\right)^{1 / 2}\left(\sum_{i=1}^{n b} r_{i}^{2}\right)^{1 / 2}}\right)
$$

where $\alpha$ is the angle between vectors and $n b$ is the number of bands in the image.

For each reference spectrum chosen in the analysis of hyperspectral images, the angle $\alpha$ is determined for every element of the image (pixel). This value in radiants is assigned to the corresponding spectrum in the output SAM image, one for each reference spectrum. The maps of the produced spectral angle show a new cube of data providing a band number equals to that of the reference spectra used for the mapping. The SAM algorithm, implemented in ENVI software, requires as an input a number of training areas or reference spectra resulting from specific "Regions Of Interest" (ROIs) or spectral databank. As far as this study is concerned, the input spectra have been extracted from ROIs accurately identified in the view, integrated with a series of spot observations of the study areas and visual analysis of additive syntheses in RGB (Red, Green, Blue), (see Figure 2). When building a GIS based on MIVIS data, the land scheme is implemented by adopting two different approaches: the vector and the raster data technique. In the raster model the representation of the objects is based on the co-ordinates (row and column) of the pixels of the matrix forming the image. In the vector model the representation of the objects is based on features: points, lines, segments, areas, polygons, etc. Once the image is classified in significant 
classes by means of supervised and/or unsupervised spectral methods, it becomes a true thematic map because the classification is based on characterization classes. The integration of the hyperspectral data in GIS (Geographic Information System) (see Figure 3), is one of the most effective applications of data integration. The morphological, chemical and physical features of the study area, can be accurately superimposed on the geographical maps to allow us to closely examine the interactions underway in the investigated or expected in the future. We would like to stress that the integration process of hyperspectral data, in the territorial information systems, has always been difficult when compared with a common remote sensing data. This depends on the nature of the employed data: in a reduced data set, there is always a loss of spectral information, relative to the original raw data.

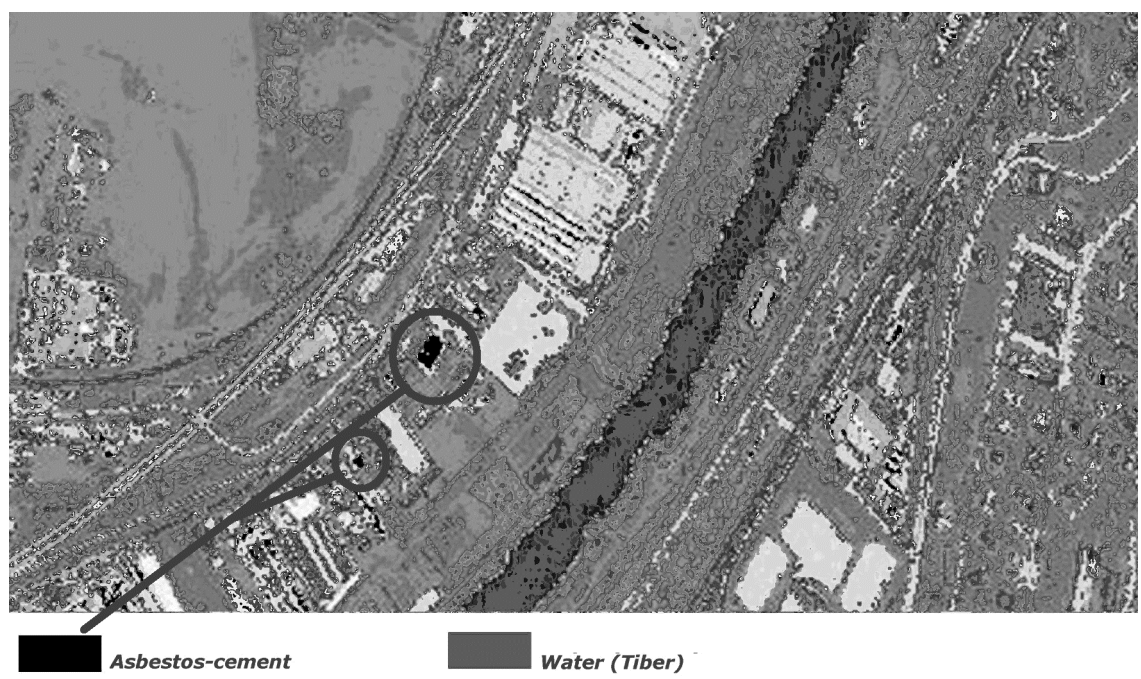

Figure 1: $\quad$ Example of brownfield classification obtained by SAM method.

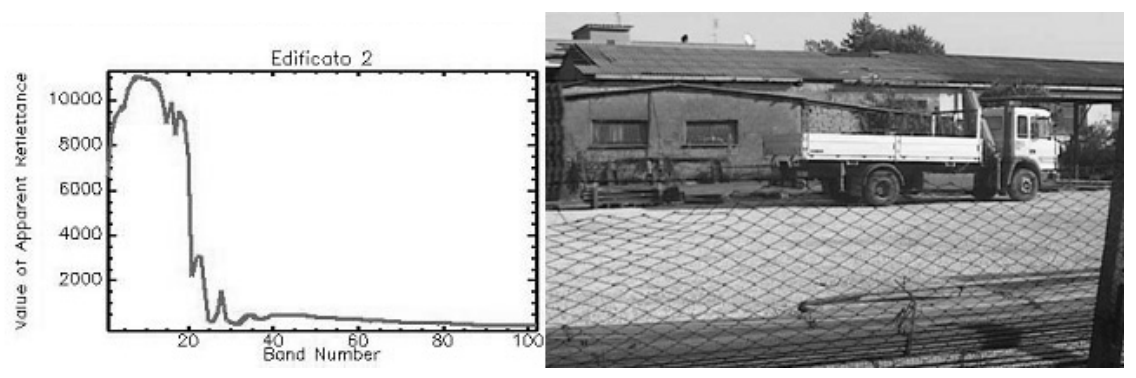

Figure 2: $\quad$ Building spectrum taken from MIVIS data, RGB image related to an urbanized area. 

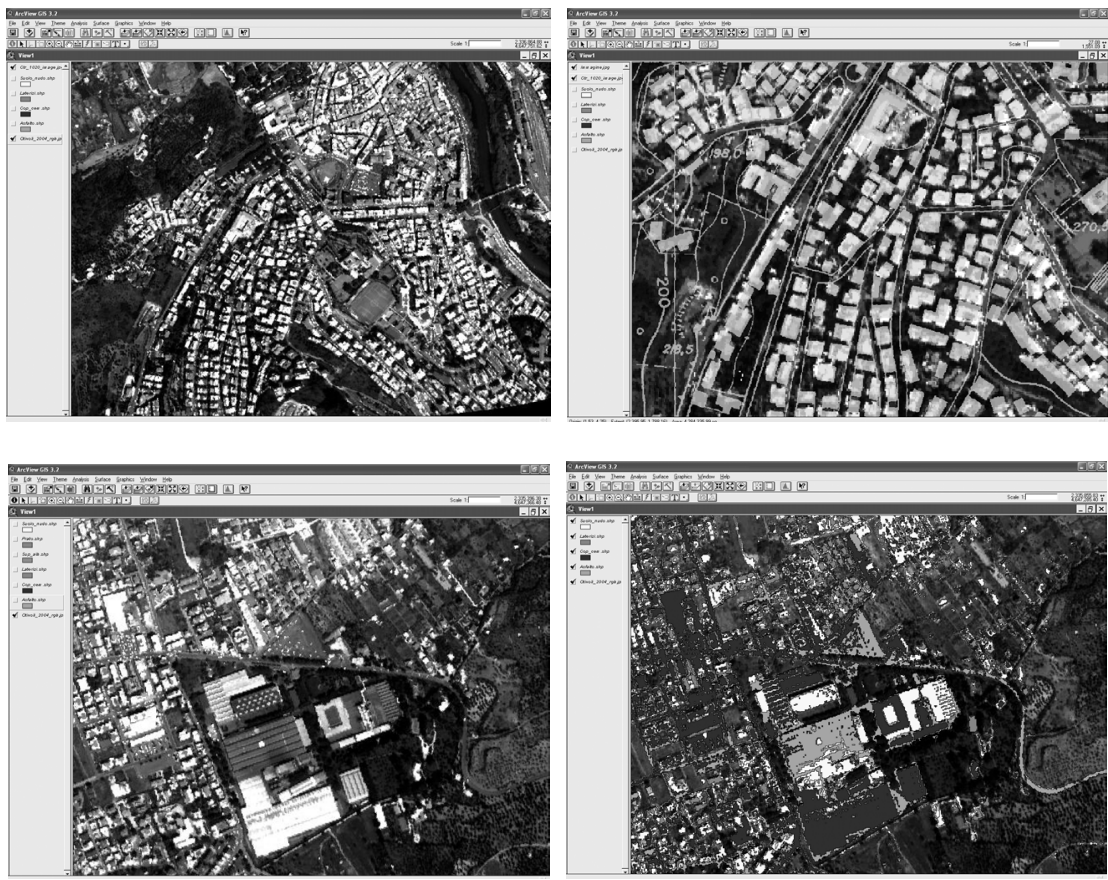

Figure 3: $\quad$ MIVIS data integration in GIS by adopting the raster and the vector models.

\section{Identification procedures: convolution and filtering techniques}

In most cases of satellite/airborne remote sensing, buildings pulled down (or abandoned) together with waste lands show irregular contours of the structure and discontinuities of the surface coverings. Detection techniques evaluate the homogeneity of lines and surfaces. In any case they will have to be optimized in terms of timing, tools and response reliability level.

Of course, we could resort to airplane campaigns intended for a software 3-dimensional reconstruction of topography, which could make easier the recognition of the wear state buildings. These campaigns are aimed at $a$ ) the DEM (Digital Elevation Model) achievement and b) a 3-D software model reconstruction of highly urbanized areas. In the former case, remote sensing systems are normally used. The new algorithmic techniques reduce the production costs of these images, obtaining, for instance by means of AsterDTM [6], a 15-m pixel resolution, mean errors on the $5 \mathrm{~m}$ altitude and final costs related to image within the range of some hundred euros. In the latter, there is the need for a more accurate elevation range, so the airplane campaigns are essentially intended for urbanized areas. About brownfields, alternative 
techniques are adopted. As previously mentioned, technicians use hyperspectral data and higher spatial resolution images, if necessary, in the Visible. An image detected in the Visible provides as database some information sensitive to geometric features, angles of the various facets whose the surface coverings is eventually composed. Continuous lines and surface homogeneity are the parameters to distinguish between a good building conservation and a highly abandoned area.

The identified procedure has used two parallel levels: the first one emphasizes the building contours, whereas the second one evaluates the indicators of the surface homogeneity level.

As far as the contours are concerned, we adopted classical filtering techniques using kernel, for example those introduced by Sobel and Prewitt (together with multi-stage process and related procedures introduced by Canny [3] and Parker [9]). Kernels operate on a pixel grid, representative of a coded image in different grey levels, and they are generally two matrices: one matrix is simply the other rotated by $90^{\circ}$. When each kernel is applied, it emphasizes the different grey levels towards the two main directions: from left/right to right/left (Sx) and from down/up to up/down (Sy). In the case of Sobel kernel, matrices are as follows:

$$
S x=\left[\begin{array}{lll}
-1 & 0 & +1 \\
-2 & 0 & +2 \\
-1 & 0 & +1
\end{array}\right] \quad S y=\left[\begin{array}{ccc}
+1 & +2 & +1 \\
0 & 0 & 0 \\
-1 & -2 & +1
\end{array}\right]
$$

The convolution operation between the matrix of the image I and the Sobel kernel provides the values related to the two matrices Gx and Gy, eqns (2) [7]. These can be normally combined together to find the absolute magnitude of the gradient, eqn (3), or in the approximated form by eqn (4) which detects the researched edge.

$$
\begin{gathered}
G x_{m, n}=\sum_{k=1}^{3} \sum_{p=1}^{3} I_{m+k-2, n+p-2} S x_{k, p} \quad ; G y_{m, n}=\sum_{k=1}^{3} \sum_{p=1}^{3} I_{m+k-2, n+p-2} S y_{k, p} \\
|G|=\sqrt{G x^{2}+G y^{2}} \\
|G|=|G x|+|G y|
\end{gathered}
$$

Before applying the convolution technique and in order to obtain results significantly useful for identifying the same edges and defining the borders, an intensity thresholding filtering is implemented. These filters can employ either fixed or adaptive threshold value. The procedure starts from the hyperspectral classification: the related maps allow one to identify the involved areas showing covering materials with possible potentially hazardous substances. These areas are geo-referred and virtually superimposed on the map referring area in the Visible (a grey-level image with a higher resolution). The different resolution between the two maps will determine a non-biunivocal correspondence: one 
pixel (hyperspectral map) $\rightarrow$ more pixel (grey-level map). In every selected area the statistics of the grey levels is evaluated. The relative pixels are aggregated into groups according to the intensity level they match. In the case of a good building conservation, histogram intensity levels consist of evident few peaks as shown in the example of Figure 4. Each group of the pixel intensity distribution produces a threshold by means of the mean value determination and the identification of a standard deviation. This filtering process is an evolution of the filtering method based on adaptive threshold value and it is mostly intended to achieve the main objective of this research activity.

The convolution operation with selected kernels outlines the boundaries among pixel with a different intensity. If we take into consideration buildings in good conditions, these boundaries should produce closed curves. But normally they could yield discontinuous lines. The number of interruptions, i.e. the absence of a regular sequence of contour, identifies an indicator of the building wear state or the lack of building walls. The above-mentioned discontinuities could be reduced by the thematic hyperspectral maps: equivalent responses in the Visible are discriminated from the different recorded qualification.
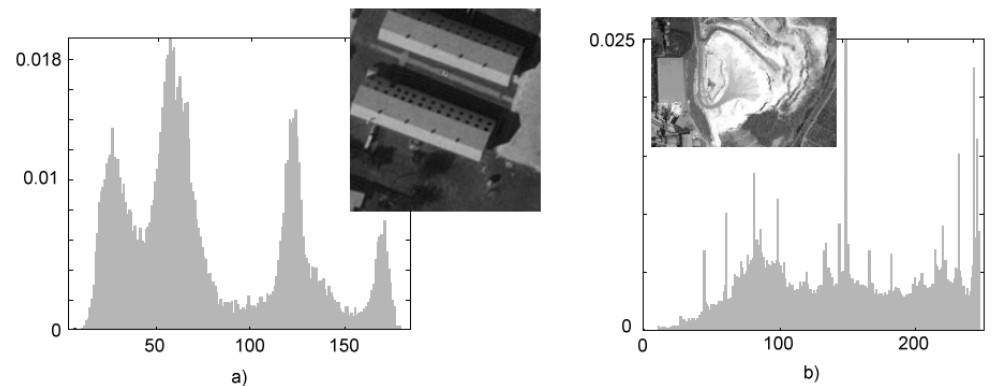

Figure 4: Two examples of histogram for typical structures: a) buildings that are in good state of conservation; b) waste lands.

Building roof mappings, for instance, differ from asphalt and grassy surfaces but, due to the colour and brightness effect, they show the same grey-level intensity. The vectorialization of the boundary sequence offers computational benefits in the following operations to verify the area to which the specific pixels belong. This transformation, however, could be burdensome and the previous operations could be replaced by raster-based equivalence classes. Together with this edge detector technique is assessed the non homogeneous levels within the study area: the non uniformity of the spatial distribution relates to covering materials such as cement, coating materials, etc. This is clearly the most costeffective procedure, if we consider the rubbish open-air depot monitoring. The non uniformity analysis can start directly from the previously mentioned thresholded filtering data or it can be evaluated by the intensity hystogram of the original image in the Visible. In particular, we get rid of operators designed to determine the different frequencies within the study area. 
The application of a FFT (Fast Fourier Transformer) provides the expected output, but, unfortunately, this method transforms the domain of the representation losing the information on the original spatial position [8]. A Wavelet transform [4, 5] allows to obtain a response which can be also located and generally applied to a more extended area. Usually, wavelet is associated to the scaling function. The last one is evaluated by convolving low-pass quadrature mirror filters, and when used in wavelet decomposition, they realize the approximation. Complementary, wavelet is valued by high-pass filter which produces the details, i.e. the discrepancy between two images generated by consecutive application of an orthogonal wavelet analysis. In the case of twodimensional context, wavelets are defined as tensor products of scaling function and three wavelets, in three orientations, are generated: horizontal, vertical and diagonal.

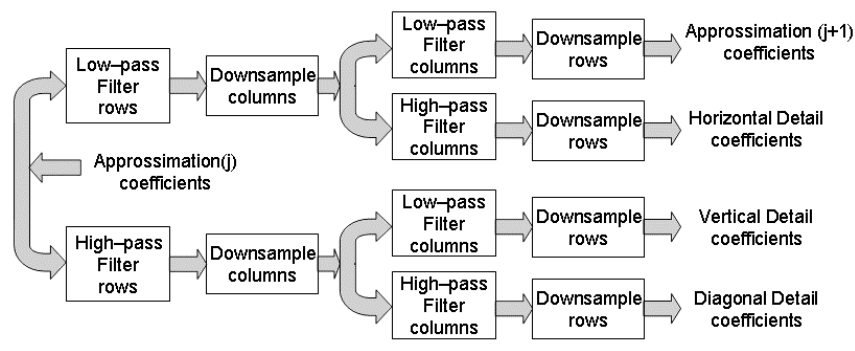

Figure 5: Wavelet: schematic algorithm of decomposition step.

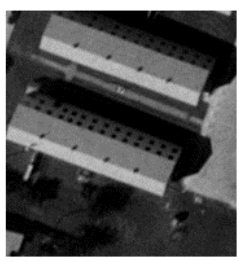

a)

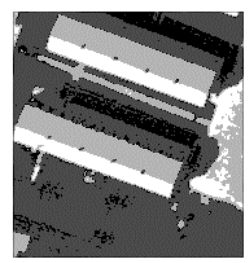

b)

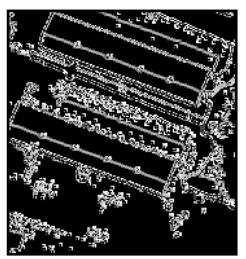

c)

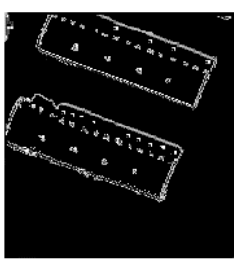

d)

Figure 6: Sequence of the building edge construction : a) Grey-level image of a block; b) after the thresholded filtering technique; c) application of the Sobel kernel convolution; d) reconstruction of discontinuous outlines and possible elimination of internal sides.

Figure 5 shows an algorithm of each decomposition step and the related dyadic decimations. Consequently, the following outputs are coarser images. Detail coefficients are localized and could help to evaluate the above-mentioned operators, designed to determine the frequencies. Figure 6 shows the sequence of the building edge construction. To the grey-level image is applied a thresholded filtering technique. Thresholds have been chosen on the local base and according to the considered materials only. If we consider the example, we can note that the match "covering material and brightness intensity in the Visible" has identified 
three thresholds which generate Figure $6 \mathrm{~b}$ ). The convolution operation with Sobel kernel produces contour sequences which are partly interrupted and unfortunately they identify objects not belonging to the study area. The relationship with the qualification data allow to isolate the considered edges only in order to reconstruct the discontinuous outlines and, if necessary, to eliminate the internal sides.

\section{Conclusions}

The results obtained within the framework of this study make foresee excellent potentialities of MIVIS hyperspectral data and GIS to monitor brownfield areas. The use of MIVIS data proved to be particularly interesting for identifying brownfield nature by means of classification techniques. Furthermore, when hyperspectral data processed in GIS are integrated, a huge potentiality of these systems is shown in integrating and correlating data from different sources to be used within the framework of a sustainable planning and development.

\section{Acknowledgements}

The authors wish to thank Mrs Alessia Turini for the English translation of the text.

\section{References}

[1] Ben-Dor, E., Kruse, F. A., Lefkoff A. B. \& Banin A., Comparison of three calibration techniques for utilization of GER 63 channel aircraft scanner data of Makhtesh Raamon Negev, Israel, $P E \& R S$, 60(11), pp.1339-1354, 1994.

[2] Boardman, J.W. \& Kruse, F.A., Automated spectral analysis: a geological example using AVIRIS data, North Grapevine Mountains, Nevada, Proc. of the Tenth Thematic Conference on Geologic Remote Sensing, San Antonio Texas USA, I:407-418, 1994.

[3] Canny, J., A computational Approach to Edge Detection, IEEE Transaction on Pattern Analysis and Machine Intelligence, 8(6), pp. 679-698, 1986.

[4] Chui, C.K., Wavelets: a tutorial in theory and applications, Academic Press., 1992.

[5] Daubechies, I., Cohen, A., Jawerth, B. \& Vial, P., Multiresolution analysis, wavelets and fast wavelet transform on an interval, $C R A S$, Paris, Ser. A, t. 316, pp. 417-421, 1993.

[6] Gatti, M., Stereoscopia ottica ASTER ed interferometria differenziale SAR a confronto, GEOmedia, GEO4all, 1, pp. 6-13, 2005.

[7] Gonzales, R. \& Woods, R., Digital Image Processing, Addison Wesley Publishing Company, 1992.

[8] Haykin, S., Adaptive Filter Theory, Prentice-Hall, Inc., 1996.

[9] Parker, J. R., Algorithms for Image Processing and Computer Vision, John Wiley \& Sons Inc., New York, pp. 23-29, 1997. 